\title{
Multiparameter segmentation and quantization of brain tumor from MRI images
}

\author{
Rajeev Ratan ${ }^{1}$, Sanjay Sharma ${ }^{2}$ and S. K. Sharma ${ }^{3}$ \\ ${ }^{1}$ Department of E \& IE, Apeejay College of Engineering, Sohna, Gurgaon, Haryana, India. \\ ${ }^{2}$ Department of ECE, Thapar University, Patiala, Punjab. India. \\ ${ }^{3}$ Department of ECE, Apeejay College of Engineering, Sohna, Gurgaon, Haryana \\ sanjay.sharma@thapar.edu
}

\begin{abstract}
In this paper, a method for segmentation of brain tumor has been developed on 2D-MRI data which allows the identification (10-15 minutes operator time) of tumor tissue with accuracy and reproducibility comparable to manual segmentation (2-6 hours operator time) making the automatic segmentation practical reality for malignant tumors. In this scheme, after a manual segmentation procedure the tumor identification has been made for the potential use of MRI data for improving the approximate brain tumor shape and 2D visualization for surgical planning.
\end{abstract}

Keywords: Brain tumor, MRI, MATLAB.

Introduction

Three dimensional segmentation is a reliable approach to achieve a proper estimation of tumor volume. The segmentation can be grouped under: Snakes (Gradient Vector Flow), Level Set Segmentation and Watershed Segmentation (Abbasi \& Mokhtarian, 2001). Among all possible methods for this purpose, watershed can be used as a powerful tool which implicitly extracts the tumor surface. Watershed segmentation uses the intensity as a parameter to segment the whole image data set. Moreover, the additional complexity of estimation imposed to such algorithms causes a tendency towards density dependent approaches (Abdel-Halim Elamy \& Maidong Hu, 2007).

The aim of this work is to design an automated tool for brain tumor quantification using MRI image data sets. This work is a small and modest part of a quite complex system. The whole system will when completed visualize the inside of the human body, and make surgeons able to perform operations inside a patient without open surgery. More specifically the aim for this work is to segment a tumor in a brain. This will make the surgeon able to see the tumor and then ease the treatment. The instruments needed for this could be ultrasound, Computer Tomography (CT Scan) and Magnetic Resonance Imaging (MRI). The technique employed for this study is Magnetic Resonance Imaging (MRI). Watershed segmentation based algorithm has been used for detection of tumor in 2D and in 3D. MATLAB software was employed to construct 2D structure of tumor. The rest of the paper is organized as follows. Section-2 presents the methodology of the problem, section 3 and 4 gives materials and implementation of the problem. The section 5 gives the results and section 6 leads to conclusion.

Research article (Rapid publication)

CIndian Society for Education and Environment (iSee)

\section{The proposed framework}

A conceptually simple supervised block-based and image-based (shape, texture, and content) technique has been used to analyze MRI brain images with relatively lower computational requirements. The process flow of our proposed methodology may be shown as Fig.1:



Fig. 1. Proposed Framework

The first section discusses how images are divided into regions using a block-based method. The second section shows how each classified block is studied individually by calculating its multiple parameter values. In this instance, the multiparameter features refer to the following three specific features: the edges $(E)$, gray values $(G)$, and local contrast $(H)$ of the pixels in the block being analyzed (Epifano \& Ayala, 2002).

Input Image

The images we got from MRI are of three types: axial Images, saggital Images, coronal Images. The number of images depend on the resolution of the movement of the MRI magnets.

\section{Preprocessing}

In preprocessing the first step is to load the MRI image data set on to the MATLAB workspace and after loading they will be processed in such a way that instead of processing 128 images in one direction a whole clip of 128 images is processed by one command, otherwise it would have been very hectic situation for processing each and every image independently. Thus after this processing there are only three clips instead of 384 separate images, i.e. one clip for axial images, one clip for saggital images and one clip for coronal images. After that all the clips

"MRI of Brain tumour" http://www.indjst.org
Rajeev Ratan et al. Indian J.Sci.Technol. 
are combined to get the single clip for further processing (Bailet et al., 1992, 1997).

\section{Multiparameter Calculations}

Recent advances in medical image analysis often include processes for an image to be segmented in terms of a few parameters and into smaller sizes or regions, to address the different aspects of analyzing images into anatomically and pathologically meaningful regions. Classifying regions using their multiparameter values makes the study of the regions of physiological and pathological interest easier and more definable. Here, multiparameter features refer to the following three specific values for the edges $(E)$, gray values $(\mathrm{G})$, and local contrast $(\mathrm{H})$ of the pixels (Nakajima et al., 1997; Bountris et al., 2005; Nacim Betrouni et al., 2007).

Edge (E) Parameter. Edge information is often used to determine the boundaries of an object. This is mainly used for analysis to derive similarity criterion for a predetermined object. The incidences of cerebral compression reduce the edge. Given this understanding, we use the Sobel edge detection method to detect image edges $\left(I_{E}\right)$ is obtained by filtering an input image with two convolution kernels concomitantly, one to detect changes in vertical contrast (hx) and the other to detect horizontal contrast (hy ), shown in equation (1). Image output ( $\left.\mathrm{I}_{E}\right)$ is obtained by calculating the gradient magnitude of each pixel, as shown in equation (2). Subsequently, the edge parameter $(E)$ is calculated, whereby $E(r, c)$ is increased by one each time when $l_{E}(x, y)={ }^{\prime}$ ' in a supervised block, as shown in equation (3)

$$
\begin{aligned}
& H_{x}=\left[\begin{array}{ccc}
-1 & 0 & 1 \\
-2 & 0 & 2 \\
-1 & 0 & 1
\end{array}\right], H_{y}=\left[\begin{array}{ccc}
-1 & -2 & -1 \\
0 & 0 & 0 \\
1 & 2 & 1
\end{array}\right] \\
& I_{E}(x, y)=\sqrt{I_{x}^{2}(x, y)+I_{y}^{2}(x, y)} \\
& E_{(r, c)}=\sum_{(x, y) \in B}\left(I E_{P}==1\right)
\end{aligned}
$$

Gray (G) Parameter. The gray parameter (G) for each block of the brain is accumulated, and controlled by a binary image $\left(I_{T}\right)$ using the $G_{D}$ value as a threshold, as shown in equation (6). $G_{D}$ value is calculated using the average pixel value (lavs) of each image slice (S) for total image slices $(T)$ of an image dataset, shown in equation (4) and (5).

$$
\begin{aligned}
& \operatorname{Iav}_{S}=\frac{1}{65536} \sum I_{p}(x, y) \\
& G_{D}=\frac{\sum_{S=0}^{T} \operatorname{Iav}_{s}}{T} \\
& G_{(r, c)}=\sum_{(x, y) \in B} I_{p}(x, y), \forall I_{T}(x, y)=1
\end{aligned}
$$

Research article (Rapid publication) OIndian Society for Education and Environment (iSee)
Vol.2 No 2 (Feb. 2009)

ISSN: 0974- 6846
The pixels intensity for each slice was calculated to establish the threshold values and thus provide the basis for analysis of clinical MR images from patients with brain tumors (Gupta \& Undril, 1995; Gering et al., 2002).

Contrast $(H)$ Parameter. Contrast $(H)$ is often used to characterize the extent of variation in pixel intensity. In the present technique, the computational program analyses the differences, especially in instances of strong dissimilarity, between entities or objects in an image I $(x, y)$.

We adopt the minimum/maximum stretch algorithm for the 8-neighborhood connectivity, where min $\mathrm{H}$ and max $\mathrm{H}$ represent the minimum and maximum intensity values of the neighborhood pixel $\mathrm{C} 8\left(\mathrm{I}_{H}\right)$, as shown in equation (7). In the previous studies, tumor cells are often associated with higher value of contrast $(\mathrm{H})$ parameter (Senaratne et al., 2006). $H_{d}$ is obtained by totaling the contrast of a supervised block, as shown in equation (8).

$$
\begin{aligned}
& I_{H}(x, y)=\left(\frac{I(x, y)-\min H}{\max H-\min H}\right) \times \max H \mid H \in C_{8}\left(I_{H}\right) \\
& H_{d(r, c)}=\sum_{(x, y) \in B} I_{H}(x, y)
\end{aligned}
$$

\section{Materials}

The computational analysis is implemented on a IBM Think Centre Pentium IV $2.80 \mathrm{GHz}$ computer with $512 \mathrm{MB}$ RAM. The support analysis software used is MATLAB and 3D Slicer. In order to evaluate the performance of our algorithms and methodology, the experiments were conducted on MRI data set.

Implementation

Preprocessing

Axial, saggital \& coronal images were loaded and viewed in the MATLAB. After this, axial, saggital \& coronal movie clips were prepared and are shown in Fig. 2.

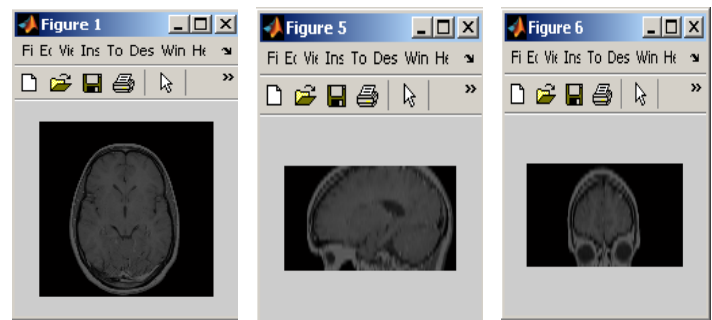

Fig. 2. Axial, saggital \& coronal movie clips

Now all the clips were combined together to produce a single clip.

\section{Multiparameter calculations}

Recent advances in medical image analysis often include processes for an image to be segmented in terms of a few parameters and into smaller sizes or regions, to address the different aspects of analyzing images into anatomically and pathologically meaningful regions. Classifying regions using their multiparameter values makes the study of the regions
Rajeev Ratan et al. Indian J.Sci.Technol. 


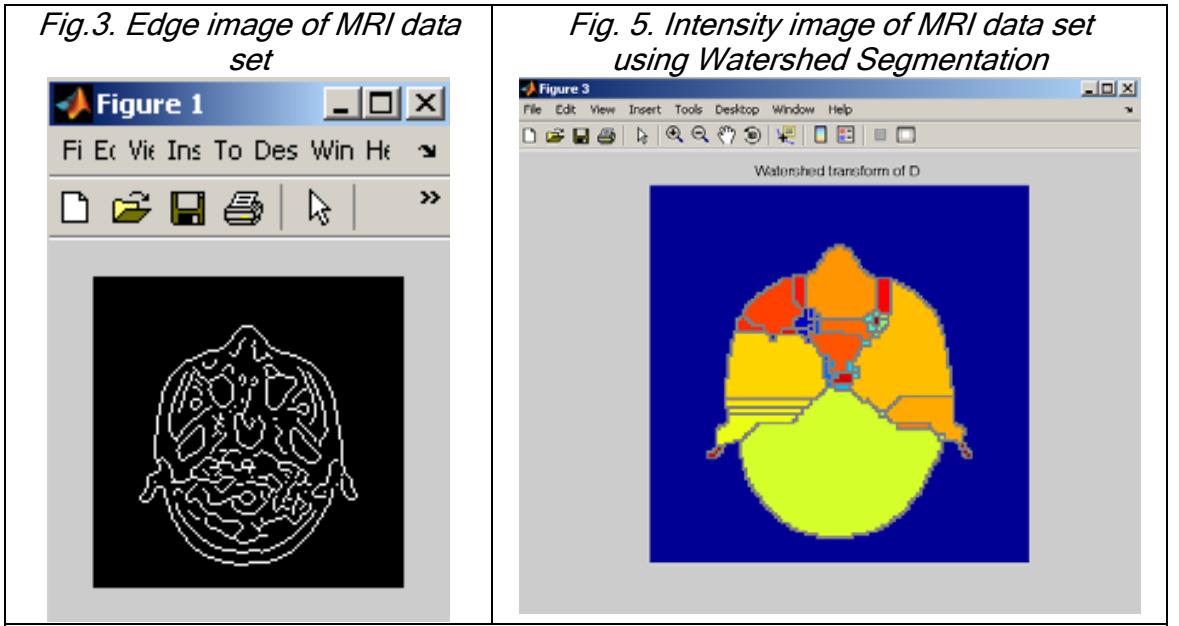

Fig. 4. Binary image of MR/ data set

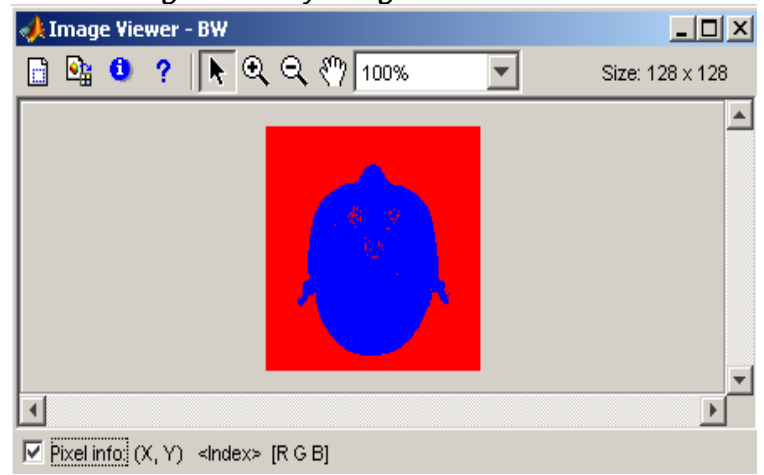

Fig.6. Constructed image after application of ROI command on MRI intensity image

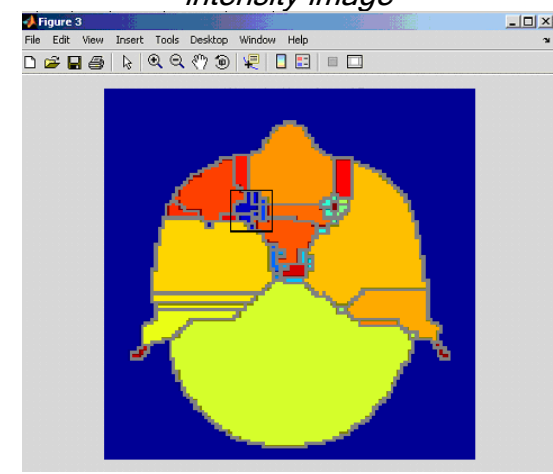

intracranial cavity. This can be achieved by executing the algorithms and the result obtained may be shown as in Fig. 3.

Gray parameter(G) calculation: The gray parameter $(G)$ for each block of the brain is accumulated, and controlled by a binary image using the value as a threshold. Pixels intensity for each slice was calculated to establish the threshold values and thus provide the basis for analysis of clinical MR images from patients with brain tumors. This can be achieved by executing the algorithm and the result obtained may be shown as in Fig. 4.

Contrast parameter $(H)$ calculation: Contrast $(\mathrm{H})$ is often used to characterize the extent of variation in pixel intensity. A computational program analyses the differences, especially in instances of strong dissimilarity, between entities or objects in an image using Watershed Segmentation. As we know that malignant tumor cells contain highly proteinaceous fluid, which is represented as high signal intensity on MRI images of the brain (Haney et al., 2001; Hinz et al., 2002) . Watershed segmentation can be used for segregating the different intensity portions and this can be achieved by executing the algorithm in MATLAB and the result obtained may be shown as in Fig.5.

Tumor Block Detection \& Visualization(2D):

Segmentation of brain tumor using Region of Interest (ROI) Command: As it has been seen from the above result that high density images have been separated from the MRI images using Watershed Segmentation. Here main aim is to segment the tumor from the MRI images. This can

of physiological and pathological interest easier and more definable. Here, multiparameter features refer to the following three specific values for the edges $(E)$, gray values $(G)$, and local contrast $(H)$ of the pixels.

Edge parameter calculation (E): Edge information is often used to determine the boundaries of an object. This is mainly used for analysis to derive similarity criterion for a pre-determined object. The reduction of edge count for tumor images is due to the fact that the substance has been pushed aside and compressed by the growth of the tumor within a confined space, the Research article (Rapid publication) CIndian Society for Education and Environment (iSee)
"MRI of Brain tumour" http://www.indjst.org
Rajeev Ratan et al. Indian J.Sci.Technol. 
executing the algorithm and the result obtained may be shown as in Fig. 8.

Results

The result of 2D in the form of pixels in $X, Y$ directions are shown in Fig. 5. As the display settings are $1028 \times 768$ pixels on the monitor of the computer and the dimensions of the monitor are $280 \mathrm{~mm} \mathrm{X}$ $210 \mathrm{~mm}$, so the dimensions of the one pixel comes out to be $0.2734 \mathrm{~mm} \times 0.2734 \mathrm{~mm}$.

Viewing the tumor from different angles the dimensions of tumor appear to be made up of seven different layers. As viewed from upside down the tumor's layer may be given the names as $1^{\text {st }}$ upper layer, $2^{\text {nd }}$ upper layer, $3^{\text {rd }}$ upper layer, middle layer, the layer below middle layer, the second last layer, the last layer. The dimensions of the different layers may be tabulated as:

\begin{tabular}{|c|c|c|c|}
\hline $\begin{array}{l}\text { Name of } \\
\text { Layer }\end{array}$ & $\begin{array}{c}\text { Dimensions } \\
\text { (Max) } \\
\text { (pixels) }\end{array}$ & $\begin{array}{c}\text { Dimensions } \\
(\mathrm{Max}) \\
(\mathrm{mm} * \mathrm{~mm}) \\
\end{array}$ & $\begin{array}{l}\text { App. Area } \\
\left(\mathrm{mm}^{2}\right)\end{array}$ \\
\hline $\begin{array}{ll}1^{\text {st }} & \text { Upper } \\
\text { Layer } & \\
\end{array}$ & $33 * 7$ & $9.02 * 1.91$ & 17.23 \\
\hline $\begin{array}{l}2^{\text {nd }} \text { Upper } \\
\text { Layer }\end{array}$ & $54 * 11$ & $14.76 * 3.01$ & 44.43 \\
\hline $\begin{array}{ll}3^{\text {rd }} & \text { Upper } \\
\text { Layer }\end{array}$ & $63 * 14$ & $17.22 * 3.83$ & 65.95 \\
\hline Middle Layer & $87 * 12$ & $23.79 * 3.28$ & 78.03 \\
\hline $\begin{array}{l}\text { Layer Below } \\
\text { the Middle }\end{array}$ & $62 * 13$ & $16.95^{*} 3.55$ & 60.17 \\
\hline $\begin{array}{l}\text { The Second } \\
\text { Last Layer }\end{array}$ & $52 * 10$ & $14.22 * 2.73$ & 38.82 \\
\hline \multirow[t]{2}{*}{$\begin{array}{ll}\text { The } & \text { Last } \\
\text { Layer } & \\
\end{array}$} & $31 * 19^{*} 6$ & $8.48 * 1.64$ & 13.91 \\
\hline & & Total & 318.54 \\
\hline
\end{tabular}

Thus we see that the total approximate area of tumor comes out to be $318.14 \mathrm{~mm}^{2}\left(3.1814 \mathrm{~cm}^{2}\right)$

\section{Conclusion}

The results show that Watershed Segmentation can successfully segment a tumor provided the parameters are set properly. The watershed method did not require an initialization while the others require an initialization inside the tumor. The visualization and quantitative evaluations of the segmentation results demonstrate the effectiveness of this approach. Watershed Segmentation algorithm performance is better for the cases where the intensity level difference between the tumor and non tumor regions is higher. It can also segment non homogenous tumors providing the non homogeneity is within the tumor region. This paper proves that methods aimed at general purpose segmentation tools in medical imaging can be used for automatic segmentation of brain tumors. The quality of the segmentation was similar to manual segmentation and will speed up segmentation in operative imaging.

Among the segmentation methods investigated, the watershed segmentation is marked out best out of all others. The user interface in the main application must be extended to allow activation of the segmentation and to collect initialization points from a pointing device and transfer them to the segmentation module. Finally the main program must receive the segmented image and present the image as an opaque volume.

\section{References}

1. Abbasi $S$ and Mokhtarian F (2001) Affine-similar shape retrieval: application to multiview 3-D object recognition. IEEE Trans. Image processing. 10 (1), 31-139.

2. Abdel-Halim Elamy and Maidong Hu (2007) Mining brain tumors \& their growth rates. IEEE Image Processing Society Journal. pp: 872-875.

3. Bailet JW, Alan Lichtenstein, Ge Chen and Robert A. Mickel (1997) Inhibition of lymphocyte function by head and neck carcinoma cell line soluble factors. Arch Otolaryngol Head Neck Surg. 123(8), 855-862.

4. Bailet JW, Abemayor E, Jabour BA, Hawkins RA, Hoh CK and Ward PH (1992) Positron emission tomogarphy: a new precise modality for detection of primary head and neck tumor and assessment of cervical adenopathy. Laryngosope. 102, 281288.

5. Black PM, Moriarty T and Alexander E (1997) Development and implementation of intraoperative magnetic resonance imaging and its neurosurgical applications. Neurosurgery. 41(4), 831-845.

6. Bountris P, Farantatos E and Apostolou N (2005) Advanced image analysis tool development for the early stage bronchial cancer detection. PWASET. 9, 151-156.

7. Deng $\mathrm{Y}$, Manjunath BS, Kenney C, Moore MS and Shin H (2001) An efficient color representation for image retrieval. IEEE Trans. Image Processing. 10 (1), 140-147.

8. Dewalle Vignion AS, Betrouni $\mathrm{N}$ and Makni $\mathrm{N}$ (2008) A new method based on both fuzzy set and possibility theories for tumor volume segmentation on PET images. $30^{\text {th }}$ Annual International IEEE EMBS Conference. 3122-3125.

9. Epifano I and Ayala G (2002) A random set view of texture classification. IEEE Trans. Image Processing. 11(8), 859-867.

10. Fadi Abu Amara and Abdel Qadar (2007) Detection of breast cancer using independent component analysis. IEEE EIT Proceedings. 236241.

11. Gering D, Eric W, Grimson L and Kikinis R (2002) Recognizing deviations from normalcy for brain tumor segmentation. Medical Image Computing and Computer-Assisted Intervention (MICCAI), Tokyo Japan.pp:388-395 (http://link.springer. de/ link/service/series/0558/bibs/2488/24880388.htm).

12. Gupta R and Undril P (1995) The use of texture analysis to delineate suspicious masses in
Research article (Rapid publication)

CIndian Society for Education and Environment (iSee)
"MRI of Brain tumour" http://www.indjst.org
Rajeev Ratan et al. Indian J.Sci.Technol. 
mammograohy. Physics in Medicine \& Biology. 40, 835-855.

13. Haney SM, Thompson PM, Cloughesy TF, Alger JR and Toga AW (2001) Tracking tumor growth rates in patients with malignant gliomas: a test of two algorithms. AJNR Am. J. Neuroradiology. 22, 73-82.

14. Hinz M, Pohle R, Shin H and Tonnies KD (2002) Region-based interactive 3D image analysis of structures in medical data by hybrid rendering. Proc. SPIE in Visualization, Image-Guided Procedures, and Display. 4681, 388-395.

15. Jason J. Corso, Eitan Sharon and Shishir Dube (2008) Efficient Multilevel Brain Tumor Segmentation with Integrated Bayesian Model Classification. IEEE Transaction on Medical Imaging. 27, 148-155.

16. Jolesz FA (1997) Image-guided procedures and the operating room of the future. Radiology. 204, 601-612.

17. Krishnan K and Atkins MS (1998) Segmentation of multiple sclerosis lesions in MRI - an image analysis approach. Proc of the SPIE Medical Imaging. 338, 1106-1116.

18. Lihua Li, Weidong $\mathrm{Xu}$, Zuobao $\mathrm{Wu}$, Angela Salem and Claudia Berman (2007) A new computerized method for missed cancer detection in screening mammography. Proc. IEEE Natnl. Conf. on Integration Technol. pp: 21-25.

19. Nacim Betrouni, Phillipe Puech and Anne Sophie Dewalle (2007) 3 D automatic segmentation and reconstruction of prostate on MRI images. Proc. $29^{\text {th }}$ Annual Intnl. Conf. IEEE EMBS. 5259-5262.

20. Nakajima S, Atsumi $\mathrm{H}$ and Bhalerao AH (1997) Computer-assisted surgical planning for cerebrovascular neurosurgery. Neurosurgery. 41, 403-409.

21. Radu Calinescu and Steve Harris (2007) Model driven architecture for cancer research. IEEE Intnl. Conf. Software Engg. Formal Methods. pp: 59-68.

22. Senaratne GG, Richard B. Keam, Winston L. Sweatman and Greame C. Wake (2006) Solution to the 2 dimensional boundary value problem for microwave breast tumor detection. IEEE Microwave \& Wireless Component Letters. 16(10), 343-348.

23. Vailaya S, Figueriodo MAT, Jain AK, and Zhang HJ (2001) Image classification for content-based Indering. IEEE Trans. Image processing. 10(1), 117-130.

24. Yao Yao (2004) Segmentation of breast cancer mass in mammograms and detection using Magnetic Resonance Imaging. IEEE Image Processing Soc. J. pp: 561-567.
"MRI of Brain tumour" http://www.indjst.org
Rajeev Ratan et al. Indian J.Sci.Technol. 